\title{
Surgical resection for bilateral giant emphysematous bullae
}

\author{
Shinsuke Kitazawa*, Yusuke Saeki, Shinji Kikuchi, Yukinobu Goto and Yukio Sato \\ Department of Thoracic Surgery, Faculty of Medicine, University of Tsukuba, Ibaraki, Japan
}

\begin{abstract}
Surgical resection is an accepted procedure for the treatment of giant emphysematous bullae. However, few studies have reported for bilateral cases. We report a case in which we unexpectedly had to perform a one-stage surgical resection of the bilateral giant bullae. A 46-year-old male presented with bilateral emphysematous giant bullae. Initially, two-stage surgery was planned in order to operate on each lung separately. Immediately following the left bullectomy, a chest radiograph showed enlargement of the contralateral giant bulla and the patient's hemodynamic status became unstable due to compression of the mediastinum by the enlarged bulla. As urgent decompression was required, a right bullectomy was promptly performed. His postoperative course was uneventful and the clinical and functional conditions were improved. On the occasion of surgical treatment of bilateral giant bullae, careful attention should be paid to the possibility of an enlargement of the contralateral bullae.
\end{abstract}

\section{Introduction}

The term 'giant bulla' refers to a bulla of the lung that occupies at least one-third of the hemithorax $[1,2]$. The most common etiology of giant bullae is cigarette smoking. Giant bullae can sometimes cause progressive dyspnea and respiratory failure. Surgical bullectomy is indicated when giant emphysematous bullae occupy over half of the hemithorax [3]. For bilateral cases, however, it remains controversial whether it is best to perform the operation at the same time for the bilateral lungs or as two-stage bullectomy. In this report, we present a case of bilateral giant emphysematous bullae in which we unexpectedly had to perform an one-stage bilateral bullectomy due to the compression of the mediastinum by the enlarged contralateral giant bulla that was caused by positive pressure ventilation during surgery.

\section{Case presentation}

A 46-year-old male was admitted to our hospital with a complaint of dyspnea on exertion(DOE). He reported smoking 2 packs of cigarettes per day for the last 30 years. Medical and family histories were unremarkable. A chest radiograph on admission showed bilateral giant emphysematous bullae occupying two-thirds of the each hemithorax (Figure 1A). Although, he had been diagnosed as having bilateral giant bulla during a medical examination performed 4 years prior, he had not undergone treatment until the occurrence of DOE. Computed tomography (CT) of the chest also revealed giant bullous lung disease and extensive pulmonary compression (Figure 1B). His vital capacity was $2410 \mathrm{~mL}$ ( $58.6 \%$ of predicted capacity) with a forced expiratory volume in 1 second $\left(\mathrm{FEV}_{1.0}\right)$ of $1430 \mathrm{~L}$ ( $41.2 \%$ of predicted volume), the $\mathrm{FEV}_{1.0} /$ FVC ratio was $62.2 \%$, and diffusing capacity of the lung for carbon monoxide (DLCO) was $13.67 \mathrm{ml} / \mathrm{min} /$ torr $(57.2 \%$ of predicted value). Surgical treatment was selected because of the increasing size of the giant bulla and worsening dyspnea. We planned to perform two-stage bilateral bullectomy with video-assisted thoracoscopic surgery (VATS), primarily because we considered simultaneous surgery for the bilateral lungs to be more invasive for the patient. He therefore underwent the left bullectomy antecedently and the operation was performed under general anesthesia, with a double-lumen endotracheal tube in place. Surgical exploration showed the broad-based giant bulla originating from the left upper lobe (Figure 2A). The bulla did not collapse despite differential ventilation. The bulla was then opened up and carefully examined from both inside and out. The giant bulla was ablated and reduced in size with a low voltage soft coagulating system. Then, the giant bulla and other small bullae were resected using an endoscopic stapling device (Figure 2B). The staple lines were covered with absorptive polyglycolide felt and fibrin glue. Immediately following the operation and before extubation, the patient's blood pressure decreased and the

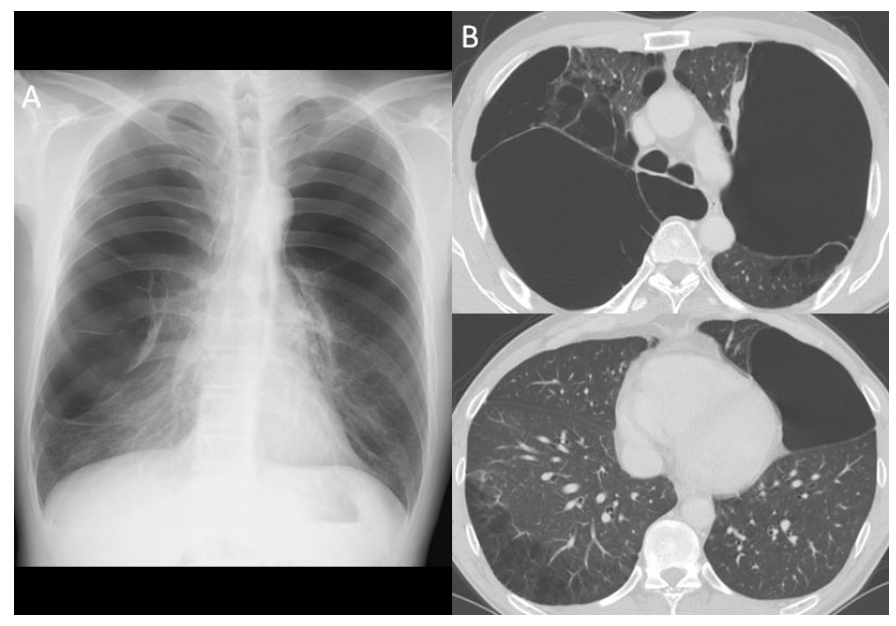

Figure 1. A) Chest radiograph on admission revealed bilateral giant emphysematous bullae. B) Chest CT on admission; despite tremendous lung compression, the nonbullous lung tissue appeared relatively well preserved in both lower lobe.

Correspondence to: Shinsuke Kitazawa, Department of Thoracic Surgery, Faculty of Medicine, University of Tsukuba, 1-1-1 Tennnodai, Tsukuba, Ibaraki 305-8575, Japan. Tel: +81-29-853-3900; Fax: +81-29-853-3904; E-mail: s1630418@u.tsukuba.ac.jp

Key words: giant emphysematous bullae, video-assisted thoracoscopic surgery (VATS), bilateral surgery

Received: February 05, 2017; Accepted: February 22, 2017; Published: February 24,2017 


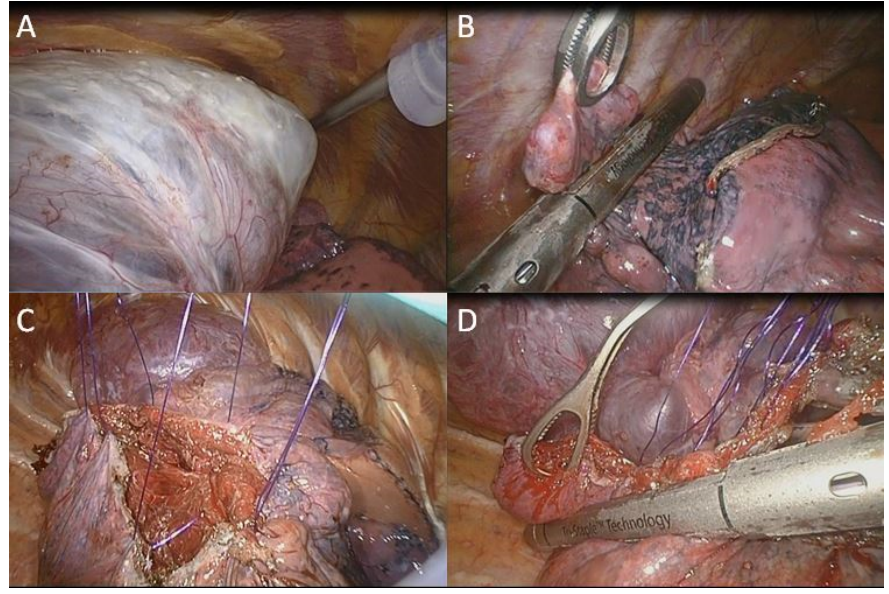

Figure 2. A) huge left upper lobe bulla had compressed the majority of the left lung. B) Other Small bullae were resected by an endoscopic stapling device. C) The right broadbased giant bulla was opened and the wall was excised down to the base. Then, simple interrupted sutures were placed through the healthy lung tissue. D) Sutured stitches were used as a traction and resected the base of the cyst using an endoscopic stapling device.

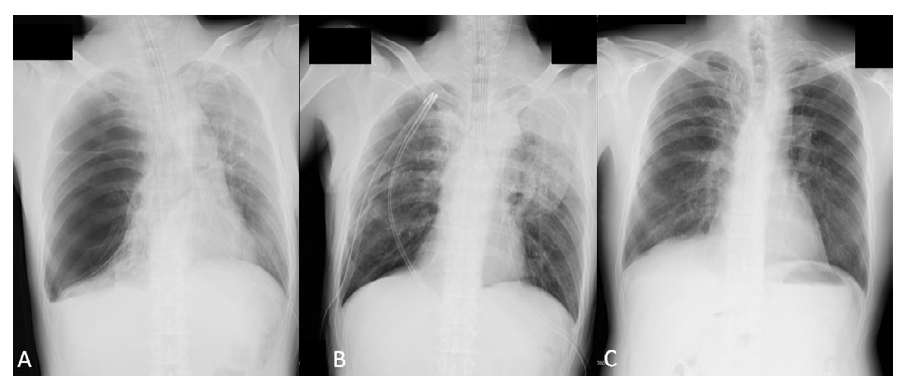

Figure 3. A) Right after the left bullectomy. Portable chest radiograph showed the significant enlargement of the contralateral bulla and the mediastinum was shifted to the left side. B) After both side of the operation. Chest X-ray revealed the expansion of the compressed lungs. C) Chest radiograph 1-year postoperatively showing complete reexpansion after giant bulla resection.

hemodynamic status became unstable. Additionally, a continuous decline was observed in both $\mathrm{PaO}_{2}$ and tidal volume. Postoperative portable chest radiograph showed a significant enlargement of the contralateral giant bulla and the mediastinum was shifted to the left (Figure 3A). We speculated that the compression of mediastinum by the enlarged right bulla had led to the unstable hemodynamic status. As urgent decompression was required, a right bullectomy was promptly performed. We approached the right pleural cavity using the same VATS technique as used on the left side. Intraoperative findings showed that the upper lobe was occupied by a giant pulmonary bulla. This broad-based giant bulla was opened and its walls were excised down to the base (Figure 2C). Rough simple interrupted sutures were then placed at the base of the cyst through healthy lung tissue. We used the sutured stitches as traction and resected the base of the cyst using an endoscopic stapling device (Figure 2D). Postoperative chest radiograph showed sufficient expansion of the bilateral lung (Figure $3 \mathrm{~B})$. He was extubated in the operating room and his postoperative course was uneventful. The bilateral chest drainage tubes were removed on postoperative day (POD) 3 and he was discharged on POD 12. Just 1 year after the operation, chest radiography showed complete reexpansion of the bilateral lungs (Figure 3C). Spirometry performed 1 -year postoperatively showed in improvement of the following measures compared with those prior to surgery: VC $3710 \mathrm{~L}(91.2 \%$ of predicted capacity); $\mathrm{FEV}_{1.0} 2510 \mathrm{~L}$ (73.2\% of predicted volume); $\mathrm{FEV}_{1.0} /$
FVC ratio $68.4 \%$; and DLCO $16.82 \mathrm{ml} / \mathrm{min} /$ torr $(71.1 \%$ of predicted value). Postoperatively, both the respiratory symptoms and pulmonary functions were significantly improved.

\section{Discussion}

The enlargement of giant bullae leads to exacerbated dispnea by compression of the surrounding normal lung parenchyma. In such cases, or when the giant bulla became infected, surgical resection is required $[4,5]$. However, the indications and operative technique for the treatment of giant bullae differ between hospitals. There are only a few case reports of the surgery, especially of bilateral cases, and it still remains controversial whether it is better to perform an one-stage bilateral surgery for both lungs or a two-stage operations to operate on each lung separately. Reports have been published on both simultaneous bilateral surgery via median sternotomy and two-stage VATS approach for bilateral giant bullae [6]. In our case, we initially selected the twostage bullectomy for the following reasons: (1) it was unclear whether the collapsed lung could re-expand with sufficient lung function after the bullectomy; (2) simultaneous bilateral surgery for both lungs seemed to be more invasive for the patient; and (3) simultaneous bilateral surgery could possibly trigger bilateral re-expansion pulmonary edema. However, immediately following the left bullectomy, a portable chest X-ray revealed compression of mediastinum by the enlarged right bulla, resulting in the patient's unstable hemodynamic status. On the occasion of surgical treatment of bilateral emphysematous giant bullae, positive pressure ventilation could causes an enlargement of contralateral bullae or a pneumothorax. Therefore, the airway pressure should be carefully kept in lower level during the surgery. In this case, the possible mechanism of the increasing size of contralaretal giant bulla was assumed that air introduced into the bulla by positive pressure ventilation was not drainaged because of a one-way valve mechanism [7]. Since the compression of a mediastinum and a functional lung worsened hemodynamic status, urgent decompression of the bulla was required and bullectomy on the right side was performed immediately. Fortunately, the patient recovered uneventfully and had reported improvement of dyspnea. In addition, there was a remarkable improvement in $\mathrm{FEV}_{1.0}, \mathrm{FEV}_{1.0} / \mathrm{FVC}$ ratio, and diffusing capacity of the lung for carbon monoxide. During the follow-up period, no new bullae appeared on the site of the bullectomy and no residual small bullae became enlarged.

In summary, on the occasion of surgical treatment of bilateral cases of giant bullae, the airway pressure should be kept as low as possible and it is important to consider the possibility of an enlargement of contralateral bullae.

\section{Conflict of interest}

The author declares no conflict of interest.

\section{References}

1. Palla A, Desideri M, Rossi G, Bardi G, Mazzantini D, et al. (2005) Elective surgery for giant bullous emphysema: a 5-year clinical and functional follow-up. Chest 128 : 2043-2050. [Crossref]

2. Tsuchida M, Nakayama K, Shinonaga M, Tatebe S, Yamaguchi A (1996) Video-assisted thoracic surgery for thorascopic resection of giant bulla. Surg Today 26: 349-352. [Crossref]

3. Meyer BF, Patterson GA (2003) Chronic obstructive pulmonary disease. 10: Bullectomy, lung volume reduction surgery, and transplantation for patients with chronic obstructive pulmonary disease. Thorax 58: 634-638. [Crossref]

4. Kayawake H, Chen F, Date H (2013) Surgical resection of a giant emphysematous bulla occupying the entire hemithorax. Eur J Cardiothorac Surg 43: e136-138. [Crossref] 
Kitazawa S (2017) Surgical resection for bilateral giant emphysematous bullae

5. Schnater JM, Plaisier PW, van den Berg PM, Schutte PR (2003) A remarkable outcome after video-assisted thoracoscopic resection of a giant bulla. Interact CardioVasc Thorac Surg 2: 589-591 [Crossref]

6. Van Natta TL, Iannettoni MD (2007) Reduction pneumoplasty for a giant right upper lobe bulla causing massive bilateral lung compression. J Thorac Cardiovasc Surg 133 : 1674-1675. [Crossref]

7. Keszler P (1988) Surgical pathology of bullae with and without pneumothorax. Eur J Cardiothorac Surg 2: 416-424. [Crossref]

Copyright: (C2017 Kitazawa S. This is an open-access article distributed under the terms of the Creative Commons Attribution License, which permits unrestricted use, distribution, and reproduction in any medium, provided the original author and source are credited. 